\title{
Cancer's fuel duel
}

\section{By Michael J. Haas, Senior Writer}

A team of Belgian and U.S. researchers has demonstrated that hypoxic tumor cells can be killed by targeting solute carrier family 16 , member 1 (monocarboxylic acid transporter 1), a membrane lactate transporter protein expressed on oxygenated tumor cells. The approach should mesh well with existing chemo- and radiotherapies, and systemic inhibition of the transporter is not likely to have severe side effects.

For more than 50 years it has been known that the tumor microenvironment consists of two distinct compartments-the oxygenated compartment, which is located near tumor blood vessels, and the hypoxic compartment, which is typically located near the tumor core. Hypoxic tumor cells are difficult to target with conventional chemo- and radiotherapies because of their low-oxygen environment and distance from blood vessels.

\section{"What's clever about MCT1 inhibition is that it targets the oxygenated tumor compartment, but its effect is on the hypoxic one." \\ -Charles Hart, \\ Threshold Pharmaceuticals Inc.}

a commercially available selective solute carrier family 16 , member 1 (monocarboxylic acid transporter 1) (SLC16A1; MCT1) inhibitor, to block MCT1 activity in squamous cervical carcinoma cell cultures. MCT1 is a membrane protein that mediates lactate uptake in human muscle cells during intense exercise.

Both methods of MCT1 inhibition completely blocked the uptake of lactate by the cancer cells.

Moreover, the lactate blockade caused the cells to switch from a balance between lactate metabolism and glycolysis to only glycolysis for energy production. This result led the team to hypothesize that forcing oxygenated cells to switch to glycolysis only in vivo might in turn cut off the glucose supply to hypoxic cells.

In mouse models of lung and colorectal cancer, inhibition of MCT1 with $\alpha$-cyano-4-hydroxycinnamate slowed tumor growth by inducing the same glycolytic switch in oxygenated tumor cells, which indeed had the added benefit of depriving hypoxic cells of glucose and killing hem.

Tumor hypoxia is associated with progression to malignancy and cancer relapse-making hypoxic tumor cells important, if elusive, drug targets.

The relationship between tumor compartments was previously thought to be strictly one way: oxygenated cells used most of the available oxygen to produce energy from glucose, leaving hypoxic cells to produce most of their energy via anaerobic fermentation, releasing lactate as a by-product.

In a paper in the Journal of Clinical Investigation, however, researchers demonstrated that the relationship is symbiotic and that the symbiosis can be exploited therapeutically. ${ }^{1}$

\section{Fuel service}

First, the team found that oxygenated human cervical squamous carcinoma cells could metabolize exogenous lactate. This led to the hypothesis that lactate released by hypoxic cells - conventionally viewed as a waste product-could actually fuel oxygenated tumor cells.

To confirm this, the team injected mice bearing squamous cervical carcinoma xenografts with ${ }^{13} \mathrm{C}$-labeled lactate, then excised the tumors 30 minutes later. NMR experiments in extracts from those tumors detected ${ }^{13} \mathrm{C}$-labeled glutamate, a lactate metabolite, which demonstrated that oxygenated tumor cells could indeed take up and metabolize lactate.

Next, the team used either RNAi or $\alpha$-cyano-4-hydroxycinnamate,
Sonveaux, an associate professor of pharmacology and therapeutics at the Université Catholique de Louvain (UCL) and lead author on the JCI paper.

The research team was led by Olivier Feron, associate professor of pharmacology and therapeutics at UCL, and Mark Dewhirst, professor of radiation oncology and pathology and biomedical engineering at Duke University Medical Center.

\section{Compartmentalized strategy}

Companies contacted by SciBX agreed that MCT1 inhibition could be a potent addition to combination therapy, but they wanted to know more about the potential side effects on muscles and neurons.

Charles Hart, VP of biology at Threshold Pharmaceuticals Inc., said the elucidation of a two-way, symbiotic interaction between tumor compartments was "a revolutionary, paradigm-shifting idea" that could lead researchers to think about cancer differently.

Although lactate transport between hypoxic and oxygenated cells in muscle tissue is well characterized, no one had thought to apply that mechanism to the tumor microenvironment-as the JCI authors did - to find out whether the oxygenated compartment was using lactate to fuel growth and proliferation, he said.

Instead, Hart said researchers were interested in how cancer cells responded to the acidifying environment that lactate caused, and 


\section{TARGETS \& MECHANISMS}

sought to take advantage of that acidic environment, for example by developing compounds that were highly membrane-permeable under acidic conditions.

"What's clever about MCT1 inhibition is that it targets the oxygenated tumor compartment, but its effect is on the hypoxic one," said Hart. Thus an MCT1 inhibitor could work well in combination with a therapy that targeted either the oxygenated or hypoxic compartment, he said.

But Hart added that the timing of dosing would be important: an MCT1 inhibitor would have to be given before any drug that was cytotoxic to oxygenated cells. Otherwise, the cytotoxic molecule could kill off the oxygenated cells before the MCT1 inhibitor could target them and kill hypoxic cells via glucose deprivation.
Hart said an MCT1 inhibitor should also combine well with other agents that selectively target the hypoxic compartment.

Threshold's TH-302 is a hypoxia-activated
"The strategy of MCT1 inhibition is clearly complementary to the strategies targeting glycolysis in tumors." -Emma Shtivelman, BioNovo Inc. cytotoxic prodrug. It is in Phase I/II trials to treat soft tissue sarcoma, solid tumors, nonsmall cell lung cancer (NSCLC) and pancreatic and prostate cancers.

Emma Shtivelman, director of cancer research at BioNovo Inc., said the JCI paper highlighted "a peculiarity of tumor cell biology" that suggested a new approach to treating cancer. "MCT1 is clearly a novel target whose inhibition could not only affect the ability of some tumor cells to take up lactate, but also disrupt glycolysis in other cells present in the same tumor," she said.

Shtivelman added that "the strategy of MCT1 inhibition is clearly complementary to the strategies targeting glycolysis in tumors,"

\section{Box 1. Un-reducing apoptosis.}

Both cancer cells and healthy neurons outlive other normal cells by deregulating mechanisms of mitochondrial apoptosis, a key step of which is the release of the molecule cytochrome $c$, somatic (CYCS) from the mitochondrial membrane in response to apoptotic stimuli such as DNA damage and reactive oxygen species. Both cell types also rely heavily on glycolysis for energy, but how that process plays a role in antiapoptosis has been unknown.

Researchers at the University of North Carolina at Chapel Hill (UNC) have now shown that glycolysis in cancer cells and neurons keeps CYCS in a reduced state, thereby preventing the protein from activating proapoptotic pathways. Based on the findings, the researchers suggested that both neurodegeneration and cancer could be treated by modulating the redox state of CYCS. ${ }^{4}$

To investigate the connection between glycolysis and antiapoptosis, the UNC group treated human cervical cancer cells, rat hepatocellular carcinoma cells and mouse embryonic fibroblasts with either hydrogen peroxide to raise the oxidation level of the cells or glutathione to lower the oxidation level. Cells treated with peroxide were more susceptible to CYCS-mediated apoptosis than cells treated with glutathione, indicating that CYCS requires an oxidizing environment to induce cell death.

Inhibition of the pentose phosphate pathway - by which glycolysis leads to glutathione production-also increased the oxidation state of the cancer cells and made them more susceptible to CYCS-mediated apoptosis.

Similarly, raising the oxidation level of healthy mouse neurons by treating them with peroxide or inhibiting the pentose phosphate pathway also promoted CYCS-mediated apoptosis.

"Our results suggest that forcing cancer cells to be in an oxidizing environment would make them more susceptible to apoptosis induced by cytochrome $c$ release from the mitochondria," thereby increasing the effectiveness of chemotherapy, Mohanish Deshmukh told SciBX. This could be accomplished by inhibiting the pentose phosphate pathway to block glutathione synthesis or by directly delivering oxidized CYCS or CYCS mimetics to the tumors, he said.

Deshmukh, an associate professor of cell and developmental biology at UNC, was an author on the Nature Cell Biology paper that described the results.

Conversely, Deshmukh said blocking oxidation of CYCS in neurons might be a strategy to treat neurodegenerative disorders, such as Parkinson's disease (PD), in which the accumulation of reactive oxygen species and oxidized proteins play a role. "Antioxidants could restrict the apoptotic pathway to spare a neuron's life," he said.

"The results are indeed very intriguing, and the link between neuronal glucose metabolism and cancer cell metabolism is appealing," said Nicolai
Savaskan, a research group leader at the University of Zurich's Institute of Brain Research.

But before the Nature Cell Biology findings could be translated into cancer therapeutics, Savaskan said, more work is needed to understand how increasing the oxidation levels of normal cells - thereby generating more reactive oxygen species in those cells-would affect their survival.

Savaskan is also a research group leader at the Swiss Federal Institute of Technology.

Deshmukh said his group has already uncovered some differences between the apoptotic pathways of normal cells and cancer cells. He cited a paper the team published last year in the Proceedings of the National Academy of Sciences, showing that the direct injection of excess CYCS induced apoptosis in brain tumor cells but not healthy neurons. ${ }^{6}$

"This opens the possibility of using cytochrome $c$ itself as a drug" to treat brain tumors, Deshmukh said. To that end, his group is looking at nanoparticles to deliver CYCS into cells and screening for small molecule mimetics of CYCS.

The group is also looking for other metabolic pathways and molecular mechanisms that are common to both cancer cells and neurons, he said.

Deshmukh said the findings reported in Nature Cell Biology are not patented. $-\mathrm{MJH}$ 
whether with glycolytic inhibitors such as 2-deoxyglucose or compounds that target enzymes in the glycolytic pathway. ${ }^{2}$

One potential strategy would be to give an MCT1 inhibitor to switch oxygenated tumor cells to glycolysis, then use a selective inhibitor of glycolysis to kill both the oxygenated and hypoxic cells, Shtivelman said.

BioNovo's BZL101 is an aqueous extract from the herb Scutellaria barbata "D. Don" of the Lamiaceae family that inhibits glycolysis and selectively induces cell death in tumors. The compound is in a Phase II trial to treat advanced breast cancer and has completed a Phase I trial in pancreatic cancer.

\section{Lactate on the mind (and muscle)}

Going forward, Threshold's Hart thought the JCI paper provided sufficient proof of concept that MCT1 is a viable drug target-assuming that additional experiments demonstrated no major adverse effects on healthy cells.

Shtivelman said the safety issue needs to be probed, because one potential drawback to MCT1 inhibition is that at least two types of normal tissues-skeletal white muscle and red muscle — can rely on lactate oxidation for energy production. She added that lactate has also been shown to play a role in the metabolic interactions between neurons and glia. ${ }^{3}$

Indeed, a paper in Nature Cell Biology by researchers at the University of North Carolina at Chapel Hill has elucidated a role for glycolysis in the antiapoptotic mechanisms of both cancer cells and healthy neurons (see Box 1, "Un-reducing apoptosis"). ${ }^{4}$

Thus, Shtivelman said, the combination of an inhibitor of lactate uptake or oxidation, plus a glycolysis inhibitor, could have dangerous effects on muscle and neuronal tissues.

UCL's Sonveaux disagreed that MCT1 inhibition could have serious toxic effects. He said MCT1 has a lower affinity for lactate than other MCTs found in healthy tissues.

"This means that MCT1 exerts a physiological role only when the other transporters are saturated-as it is the case, for example, during intensive exercise" or recovery from ischemic injuries, Sonveaux said.

Sonveaux also noted that patients with exercise intolerance due to ubiquitous MCT1 deletion have no symptoms while at rest. "These patients experience muscle cramps only during intensive exercise, and the symptoms are reversible," he said.

Similarly, he noted that whereas both MCT1 and MCT2 can facilitate lactate transport in neurons, MCT2 has the higher lactate affinity of the two, suggesting that the effects of MCT1 inhibition on neurons might be limited to transient lethargy.

Finally, Sonveaux said the team that published the JCI paper observed no pathological symptoms or gross behavioral changes in mice given $\alpha$ cyano-4-hydroxycinnamate.

\section{Fuel speed ahead}

Sonveaux added that other known MCT1 inhibitors have off-target effects due to poor selectivity for MCT1. But despite its selectivity for MCT1, Sonveaux said $\alpha$-cyano- 4 -hydroxycinnamate was not a drug candidate because it is poorly soluble in water. The team is collaborating with chemists to develop more drug-like analogs, he said.

The team is also designing high throughput screens to identify new MCT1 inhibitors.
Sonveaux and Feron declined to disclose the IP status of their findings. Sonveaux did say the team was seeking to collaborate with at least two undisclosed companies that already have MCT1 inhibitors in discovery for noncancer indications.

Meanwhile, Dewhirst and colleagues at Duke Medical Center have found genetic correlations between the in vitro responses of cancer cells to lactate accumulation and clinical outcomes in vivo.

In a report in PLoS Genetics, the Duke team showed that human mammary epithelial cells can respond to the accumulation of extracellular lactate (lactic acidosis) by shutting down glycolysis-a negative-feedback loop that minimized the extent of glycolysis and hypoxia in those tumors. ${ }^{5}$

The genetic markers associated with a strong response to lactic acidosis were also associated with positive clinical outcomes in breast cancer patients. This led the team to write that those genetic markers could be prognostic indicators for breast and possibly other cancers.

The findings could lead to new therapeutic strategies to treat cancer, according to Jen-Tsan Ashley Chi, leader of the PLoS research team and assistant professor of molecular genetics and microbiology at Duke University Medical Center.

"The cancer cell has a way of sensing the $\mathrm{pH}$ outside itself and adjusting its lactate production accordingly," he said.

The group is investigating the molecular and transcriptional mechanisms by which the cell accomplishes this balancing act.

A small molecule that triggered the same response to lactic acidosis-even in the absence of extracellular lactate-could be one possible therapeutic application of those ongoing studies, said Chi.

Coauthor Dewhirst said the findings reported in PLoS Genetics are not patented.

Haas, M.J. SciBX 1(45); doi:10.1038/scibx.2008.1091

Published online Dec. 18, 2008

\section{REFERENCES}

1. Sonveaux, P. et al. J. Clin. Invest.; published online Nov. 20, 2008; doi:10.1172/JCl36843

Contact: Pierre Sonveaux, Université Catholique de Louvain, Brussels, Belgium e-mail: pierre.sonveaux@uclouvain.be

2. Pelicano, H. et al. Oncogene 25, 4633-4646 (2006)

3. Hashimoto, T. et al. PLoS One 3, e2915; published online Aug. 13, 2008; doi:10.1371/journal.pone.0002915

4. Vaughn, A. \& Deshmukh, M. Nat. Cell Biol.; published online Nov. 23, 2008; doi:10.1038/ncb1807

Contact: Mohanish Deshmukh, University of North Carolina at Chapel Hill, Chapel Hill, N.C.

e-mail: mohanish@med.unc.edu

5. Chen, J.L.-Y. et al. PLoS Genet. 4, e1000293; published online Dec. 5, 2008; doi:10.1371/journal.pgen.1000293

6. Johnson, C. et al. Proc. Natl. Acad. Sci. USA 104, 20820-20825 (2007)

COMPANIES AND INSTITUTIONS MENTIONED

BioNovo Inc. (NASDAQ:BNVI), Emeryville, Calif.

Duke University Medical Center, Durham, N.C.

Swiss Federal Institute of Technology, Zurich, Switzerland

Threshold Pharmaceuticals Inc. (NASDAQ:THLD), Redwood City, Calif.

Université Catholique de Louvain, Brussels, Belgium

University of North Carolina at Chapel Hill, Chapel Hill, N.C.

University of Zurich, Zurich, Switzerland 